4. C. Kuratowski and W. Sierpinski, Les fonctions de classe 1 et les ensembles connexes ponctiformes, Fund. Math. vol. 3 (1922) pp. 303-313.

5. M. E. Munroe, Measure and integration, Cambridge, Addison-Wesley, 1953.

6. C. H. Rowe, Note on a pair of properties which characterize continuous functions, Bull. Amer. Math. Soc. vol. 32 (1926) pp. 285-287.

7. T. Tanaka, On the family of connected subsets and topology of spaces, Journal of the Mathematical Society of Japan, vol. 7, 1955.

8. R. Vaidyanathaswamy, Treatise on set topology, Madras, 1947.

9. L. Vietoris, Stetige Mengen, Monatschefte fur Mathematik und Physik vol. 31 (1921) pp. 173-204.

10. O. H. Hamilton, Fixed points for certain noncontinuous transformations, Proc. Amer. Math. Soc. vol. 8 (1957) pp. 750-756.

University of Pittsburgh

\title{
A MULTIPLICATION THEOREM FOR POSITIVE REAL FUNCTIONS ${ }^{1}$
}

\author{
F. M. REZA ${ }^{2}$
}

Modern network synthesis relies heavily on the use of Positive Real Functions. A function $Z(s)$ of the complex frequency $s$ is said to be a P.R.F. (short for Positive Real Function) when it satisfies the following three conditions.

(a) $Z(s)$ is analytic and single valued for $\operatorname{Re} s \geqq 0$ except possibly for poles on the imaginary axis,

(b) $Z(s)$ is real for real $s$,

(c) $\operatorname{Re} Z(s) \geqq 0$ for $\operatorname{Re} s \geqq 0$.

When in addition $\operatorname{Re} Z(i y)=0$, the function $Z(s)$ will be called an I.P.R.F.

The object of this note is to contribute two theorems clarifying the "algebra" of Positive Real Functions, and providing a key for their generation. These theorems employ a simple normalization procedure (operation $N$ ) after which the product of any number of normalized P.R.F.'s is a normalized P.R.F. Since obviously the sum of several P.R.F.'s is a P.R.F. then one will obtain a rather simple algebra for these functions. One may symbolically write:

Received by the editors May 20, 1957 and, in revised form, June 26, 1957 and November 2, 1957.

1 This paper was written in the course of research conducted under the sponsorship of the Office of Ordnance Research, U. S. Army.

2 This paper was presented to the International Scientific Radio Union's twelf th General Assembly, Boulder, Colorado, 1957. 


$$
\begin{gathered}
\sum \text { P.R.F. = P.R.F., } \\
N^{-1}\left\{\prod[N(\text { P.R.F. })]\right\}=\text { P.R.F. }
\end{gathered}
$$

The symbols $\sum$ and $\Pi$ stand for sum and product respectively. $N$ stands for the operation which will be described below. The consequences of these basic theorems are broad. Corollaries of these theorems include some of the results previously obtained on the behavior of electrical networks in $[1 ; 2 ; 3 ; 4]$.

Theorem 1. Let $z_{1}, z_{2}, \cdots, z_{k}, \cdots, z_{n}$ be P.R.F. Then:

$$
Z=\frac{\prod_{1}^{n}\left(1+z_{k}\right)-\prod_{1}^{n}\left(1-z_{k}\right)}{\prod_{1}^{n}\left(1+z_{k}\right)+\prod_{1}^{n}\left(1-z_{k}\right)}
$$

is a P.R.F

Proof. For $\operatorname{Re} s \geqq 0$ we have by definition $\operatorname{Re} z_{k} \geqq 0$. The transformation:

$$
(\text { Operation } N) \quad w_{k}=\frac{1-z_{k}}{1+z_{k}}=N\left(z_{k}\right)
$$

maps the right half of the $z_{k}$-plane onto the unit disc of the $w_{k}$-plane. Now consider the function:

$$
W=\prod_{1}^{n} w_{k}
$$

For a point of the $s$-plane with $\operatorname{Re} s \geqq 0$ one obtains a corresponding image point in the $W$-plane. The latter point will necessarily lie within or on the unit circle. This because the product of several numbers all in or on the unit circle cannot be outside of the unit circle. The transformation:

$$
Z=\frac{1-W}{1+W}
$$

maps the unit circle of the $W$-plane onto the right half of the $Z$-plane. Thus $Z(s)$ satisfies conditions (a), (b) and (c) and so is a P.R.F.

$$
Z(s)=\frac{1-\prod_{1}^{n} w_{k}}{1+\prod_{1}^{n} w_{k}}=\frac{\prod_{1}^{n}\left(1+z_{k}\right)-\prod_{1}^{n}\left(1-z_{k}\right)}{\prod_{1}^{n}\left(1+z_{k}\right)+\prod_{1}^{n}\left(1-z_{k}\right)} .
$$


Corollary 1. Let $z_{k}, k=1,2, \cdots, n$, be I.P.R.F.'s then $Z(s)$ as described in (3) will also be an I.P.R.F.

Proof. $z_{k}$ will map the axis of imaginaries of the $s$-plane onto the axis of imaginaries of the $z_{k}$-plane and the unit circle of the $w_{k}$-plane. This will lead in turn to the unit circle of the $W$-plane for the corresponding map of $\operatorname{Re} s=0$ by the $W$ function. Finally the unit circle of the $W$-plane will be mapped onto the axis of imaginaries of the $Z$ plane.

An Inverse Theorem. We have proved that the product of normalized P.R.F.'s in the above sense is a normalized P.R.F. The inverse of the operation (3) has not yet been discussed. For simplicity consider the case of two P.R.F.'s $z_{1}$ and $z_{2}$. On the basis of the preceding theorem

$$
Z=\frac{\left(1+z_{1}\right)\left(1+z_{2}\right)-\left(1-z_{1}\right)\left(1-z_{2}\right)}{\left(1+z_{1}\right)\left(1+z_{2}\right)+\left(1-z_{1}\right)\left(1+z_{2}\right)}=\frac{z_{1}+z_{2}}{1+z_{1} z_{2}}
$$

is a P.R.F. Now the question is to see whether for a given pair of P.R.F.'s, say $Z$ and $z_{2}$, one can always find a P.R.F. $z_{1}$ such that (9) is satisfied. Let

$$
z_{1}=\frac{Z-z_{2}}{1-z_{2} Z}
$$

Apply transformation (4) to $z_{1}$ :

$$
\frac{1-z_{1}}{1+z_{1}}=\frac{1-\frac{Z-z_{2}}{1-z_{2} Z}}{1+\frac{Z-z_{2}}{1-z_{2} Z}}=\frac{(1-Z)\left(1+z_{2}\right)}{(1+Z)\left(1-z_{2}\right)},
$$

$W$ and $w_{2}$ are by definition confined to the unit circle. $w_{1}$ will not necessarily be generally confined to the unit circle. The condition under which this is possible is given by:

$$
\left|W \cdot w_{2}^{-1}\right| \leqq 1 \quad \text { for } \operatorname{Re} s \geqq 0 .
$$

Note that if $z_{2}$ is an I.P.R.F. then

$$
\left|w_{2}\right|=\left|w_{2}^{-1}\right|=1 \quad \text { for } \operatorname{Re} s=0 .
$$

If all poles and zeros of $w_{2}$ coincide respectively with some of the zeros and poles of $w$, then $w_{1}$ will be less complex than $w$. This will 
in turn lead to a less complex P.R.F. when the condition (12) is satisfied.

COROllaRY 2. Let $z_{2}$ be an I.P.R.F., $Z$ a general P.R.F. then $z_{1}$ also is a P.R.F., provided (12) is satisfied and $Z z_{2} \neq 1$,

$$
z_{1}=\frac{Z-z_{2}}{1-z_{2} Z}
$$

THEOREM 2. Let $a_{k}, k=0,1,2, \cdots, n$ be real numbers such that $\left|a_{k}\right| \leqq 1$, let $Z$ be any P.R.F., and let

$$
P(N Z)=\frac{1}{n+1}\left[a_{0}(N Z)^{n}+a_{1}(N Z)^{n-1}+\cdots+a_{n-1}(N Z)+a_{n}\right] .
$$

Then the normalized polynomial $N[P(N Z)]$ will also be a P.R.F.

This theorem may be readily proved, and the proof will be omitted here.

In the light of the preceding theorems one can easily establish a variety of corollaries pertinent to the behavior of P.R.F.

\section{REFERENCES}

1. P. I. Richards, $A$ special class of functions with positive real part in a half-plane, Duke Math. J. vol. 14 (1947) pp. 777-786.

2. R. Bott and R. J. Duffin, Synthesis without ideal transformers, Journal of Applied Physics vol. 20 (1949) p. 816.

3. F. M. Reza, A supplement to the Brune synthesis, AIEE Communication and Electronics, Number 17, 1955, pp. 85-90.

4. A. Fialkow and I. Gerst, Impedance synthesis without minimization, Journal of Mathematics and Physics vol. 39 (1955) pp. 160-168.

SyRACUSE UNIVERSITY 